\title{
From stretched horizon to observables of quark-gluon plasma
}

\author{
V.I. Zakharov* \\ Institute of Theoretical and Experimental Physics, \\ B. Cheremushikinskaya, 25, Moscow; \\ Moscow Institute of Physics and Technology, \\ Dolgoprudny, Moskovskaya oblast 141700 \\ E-mail: vzakharoveitep.ru \\ H. Verschelde \\ Department of Physics and Astronomy, University of Ghent \\ Krijgslaan 281, Gent, Belgium \\ E-mail: henri.verscheldeeughent.be
}

\begin{abstract}
We consider non-perturbative effects in Yang-Mills theories in the deconfining phase, $T>T_{c}$. Such effects might determine large-distance physics of the quark-gluon plasma. The basic theoretical tool is the use of the dual models for large- $N_{c}$ Yang-Mills theories. Large-distance physics corresponds to the physics of the stretched horizon in the dual models. We consider nonperturbative effects at $T>T_{c}$ in parallel with the much more commonly known case of the confining phase, or $T=0$. We start with an analogue to instantons which turns to be $3 \mathrm{~d}$ (spatial dimensions) point-like defects. These high-temperature defects are not related to the topological charge and their signature is not so clean as that of instantons. Nevertheless, they contribute to the pressure and energy density of the plasma in a well deifned way, and this prediction can be tested on the lattice. There are also more complicated defects which are analogues of the magnetic strings of the confining phase. In this case, the signature of the lower-dimensional defects in the deconfining phase is similar to that of the thermal scalar.
\end{abstract}

Xth Quark Confinement and the Hadron Spectrum,

October 8-12, 2012

TUM Campus Garching, Munich, Germany

\footnotetext{
* Speaker.
} 


\section{Introduction}

Holographic models represent an alternative approach to non-perturbative, or large-coupling theories. In case of Yang-Mills theories, either pure or with quarks, no dual models were actually found, however. The best achievement remains the model [?, ?] which belongs in the infrared to the same universality class as the large- $N_{c}$ Yang-Mills theories. The geometry of the model at temperature $T=0$ is fixed as:

$$
\begin{array}{r}
d s^{2}=\left(\frac{u}{R}\right)^{3 / 2}\left(-d t^{2}+\delta_{i j} d x^{i} d x^{j}+f(u) d x_{4}^{2}\right)+\left(\frac{u}{R}\right)^{3 / 2}\left(\frac{d u^{2}}{f(u)}+u^{2} d \Omega_{4}^{2}\right), \\
\text { where } f(u)=1-\left(\frac{u_{\Lambda}}{u}\right)^{3}, x_{4} \sim x_{4}+\beta_{4}, \beta_{4}=\frac{4 \pi}{3}\left(\frac{R^{3}}{u_{\Lambda}}\right) .
\end{array}
$$

Here $u$ is an extra coordinate, $\infty>u>u_{\Lambda}$, which is conjugate to the momentum transfer, or resolution of measurements in the standard $4 \mathrm{~d}$ space. The flux through the $4 \mathrm{~d}$ unit sphere, $d \Omega_{4}$, is holographically related to the baryonic charge and will not concern us here. The limit $u \rightarrow \infty$ corresponds to the physics in the ultraviolet, or measurements with perfect resolution. One would expect to find the standard $4 \mathrm{~d}$ space on this boundary, $u=\infty$. This is not true, however, in the model considered. Instead, there lives on the boundary a $5 \mathrm{~d}$ space, which is the ordinary Minkowski space plus an extra compact coordinate, $x_{4}$, with periodicity denoted as $\beta_{4}$. The parameter $\beta_{4}$ is of order of the hadronic scale, $\beta_{4} \sim \Lambda_{Q C D}^{-1}$. The presence of this, unphysical coordinate limits the applicability of the model to large distances, $r_{\text {hydro }}$,

$$
r_{\text {hydro }} \gg \Lambda_{Q C D}^{-1}
$$

This means that, as far as the hadrons are concerned, only the pion physics can be consistently considered at small temperatures [?]. Another, much less emphasized application of the model (??) is the theory of vacuum defects, or condensates, for examples and review see [?, ?, ?]. Geometrically, the lower-dimensional defects correspond to branes wrapped around at least one of the compact coordinates. Such defects are topologically stable as far as the radius of the corresponding compact coordinate does not vanish. If it does vanish then the corresponding defects are not suppressed by large $N_{c}$ and might condense. In these two cases, pions and vacuum defects, when the model (??) is expected to apply, it does turn to be a success. Also, due to the presence of the horizon at $u=u_{\Lambda}$, the model incorporates the confinement.

One of the crucial points is that the wrapping $n_{x_{4}}$ times around the $x_{4}$-circle implies a nontrivial topological charge associated with the defect,

$$
Q_{t o p}= \pm n_{x_{4}}
$$

where the sign depends on the direction of the wrapping. The topological charge here is the standard one: $Q_{t o p}=g^{2} / 16 \pi^{2} \int d^{4} x\left(G_{\mu \nu}^{a} \tilde{G}_{\mu \nu}^{a}\right)$, where $G_{\mu \nu}^{a}$ is the field-strength tensor of the gluon field.

At the temperatures above the deconfinement phase transition, $T>T_{c}$, one deals with the plasma. As is by now well known, for a review see, e.g., [?] the quark-gluon matter at $T>T_{c}$ is well described by hydrodynamics and one can talk about the strongly interacting quark-gluon 
plasma. Remarkably enough, the limit (??) becomes the hydrodynamic limit. Thus, hydrodynamics is the main focus of our discussion. Note that the geometry (??) is to be modified at high temperatures. First, usually one is considering the Euclidean version of the model, with the compact time direction $\tau: \tau \sim \tau+1 / T$. Moreover-and this point turns to be crucial-the position of the horizon is no longer fixed at $u_{h}=u_{\Lambda}$ but is inverse proportional to the temperature, $u_{h} \sim 1 / T$. The deconfinement phase transition happens at the point $\beta_{4}=1 / T$ and is viewed in the dual models as the Hawking-Page transition of the general relativity [?].

In the next sections we will consider consequences from the dual model introduced above for the non-perturbative effects in Yang-Mills plasma (presumably, adding quarks does not change the basic properties of the plasma). This talk is partly based on the papers [?].

\section{Three-dimensional "thermal instantons"}

In the language of the dual models, the BPST instantons of the Yang-Mills theory are identified with D0 branes,- which are one-dimensional objects,- wrapped once around the compact $x_{4}$-dimension [?]. The action associated with the instantons is proportional to the radius of the $x_{4}$-circle,

$$
S_{\text {instanton }} \sim T_{1} \cdot R_{x_{4}}
$$

where $T_{1}$ is the tension of the $D 0$ brane. The crucial point is that according to the model (??) the radius $R_{x_{4}}$ vanishes on the horizon, $u=u_{h}$ :

$$
\lim _{u \rightarrow u_{h}} R_{x_{4}}=0, \quad T<T_{c} .
$$

Eq. (??) implies that the instanton action vanishes on the horizon, or in the far-infrared.

In the standard field-theoretic language instantons are specified through the position of the center, $x_{0}$ and size $\rho$. The $D 0$ branes are point-like defects in ordinary $4 \mathrm{~d}$ space and their position can be identified with $x_{0}$. A $D 0$ branes has a particular value of the coordinate $u$ as well. As is mentioned above, the value of the coordinate $u$ is associated with the resolution, or smearing in the ordinary space. Tending $u \rightarrow \infty$ corresponds to the instanton size $\rho \rightarrow 0$, while tending $u \rightarrow u_{\Lambda}$ corresponds to $\rho \rightarrow \infty$. Thus, vanishing of the action of the $D 0$ branes on the horizon, exhibited by the Eqs. (??,??) does agree qualitatively with the standard field-theory result according to which the instanton action is given by $S_{\text {instanton }}=8 \pi^{2} / g^{2}(\rho)$ and, indeed, vanishes in the limit of strong coupling, or size $\rho$ being large.

Consider now the deconfining phase, or $T>T_{c}$. In this case the dual model considered contains a black brane (generalization of black hole to higher dimensions) situated at $u=u_{h} \sim 1 / T$. Moreover,

$$
\lim _{u \rightarrow u_{h}} R_{\tau}(u)=0, R_{x_{4}}(u)=\text { const }, \quad T>T_{c},
$$

where $R_{\tau}$ is the radius of the Euclidean time coordinate. Now, the $D 0$ brane wrapped around the $\tau$-direction has a vanishing action in the limit of coordinate $u$ approaching the horizon, $u \rightarrow u_{h}$ [?]. We could call the corresponding defect as "thermal instanton". Periodicity of the defect in the $\tau$ coordinate means that we have a static defect, depending on spatial coordinates $\mathbf{x}$. 
Unlike the case of the ordinary instantons, there is no immediate candidate for the "thermal instanton" in terms of field theory. There does exist, however, a well-known defect, the Polyakov line,

$$
L(\mathbf{x})=\frac{1}{N_{c}} \operatorname{Tr}\left(\mathscr{P} \exp i g \int_{0}^{1 / T} A_{0}(\mathbf{x}, \tau) d \tau\right),
$$

which shares some properties of the conjectured "thermal instanton". Indeed, the Polyakov line (??) depends only on the spatial coordinates. Moreover, it is widely speculated that in the deconfining phase the vacuum expectation value

$$
\langle L\rangle \neq 0
$$

see, e.g., [?] and references therein. The main difference of the defect (??) from the "thermal instanton" is that in the latter case the direction of the wrapping around the Euclidean time matters. We can suggest the following analogy. For ordinary (anti)instantons there are two different solutions corresponding to $Q_{t o p}= \pm 1$, or $G^{2}= \pm G \tilde{G}$. However, if we concentrate on vacuum properties then instantons generate $<G^{2}>_{\text {instantons }} \neq 0$ while $<G \tilde{G}>_{\text {instantons }}=0$. Similarly, the non-vanishing expectation value

$$
<L>_{\text {thermal instanons }} \neq 0
$$

can be generated by the thermal instanstons although the value of $L$ is not sensitive to the direction of the wrapping around the Euclidean time coordinate.

\section{Nonperturbative pressure and energy density}

Let us start again with the instanton example. As is mentioned above, instantons generate $<G^{2}>_{\text {instantons }} \neq 0$. On the other hand, $G^{2}$ is proportional to the action density of the gluon fields. Thus, instantons are generating non-perturbative action density, or cosmological constant, finite at that. The crucial point is that this action is negative in the Euclidean time

$$
S_{\text {non-perturbative }}=-(\text { const }) \int d^{4} x,
$$

where the constant in the r.h.s. is positive definite [?]. Note also that this observation concerning the sign applies actually to any non-perturbative fluctuations, not only to the instantons. The reason for the negative sign is that the pure perturbative vacuum has action larger than the true vacuum, which includes the non-perturbative contributions as well. The non-perturbative effects are so to say "always present", and this is the physical meaning of the negative action.

Generalizing to the case of the deconfinement, we have static non-perturbative defects [?, ?]. They do generate non-perturbative action density corresponding to $3 \mathrm{~d}$ branes in $4 \mathrm{~d}$ space:

$$
<T_{\mu \nu}>_{\text {non-pert }} \sim(0,-p,-p,-p), T>T_{c},
$$

where the constant $p$ is positive and depends on a particular model of the non-perturbative physics. Another formulation is that the trace of the energy-momentum tensor generated non-perturbatively is negative-definite:

$$
<T_{\mu \mu}>_{\text {non-perturbative }}<0 .
$$

Note that there is strong support for the validity of (??) coming from the lattice measurements [?]. 


\section{Thermal $D 2$ branes}

Apart from $D 0$ branes one should consider defects of other dimensions. In particular, it has been established that in the confining phase, $T=0$, so called magnetic strings play the central role $[?, ?, ?]$. The magnetic strings are three-dimensional branes, once wrapped around the compact $x_{4}$ direction and having two non-compactified directions, which means that they represent strings in the ordinary $4 \mathrm{~d}$ Euclidean space and are topologically charged. These branes have also a vanishing action in the infrared, due to the same geometric property (??).

Similarly, we expect $D 2$ branes wrapped around the Euclidean compact time coordinate to be unsuppressed by the action in the thermal vacuum at $T>T_{c}$ [?]. There are two types of such branes. First, we may have $D 2$ branes with two non-compact spatial coordinates. From the point of view of $3 \mathrm{~d}$ space these defects would look like strings. Moreover $2 \mathrm{~d}$ defects in $3 \mathrm{~d}$ space are equivalent to $1 \mathrm{~d}$ defects (on the dual lattice). The $1 \mathrm{~d}$ defects, or lines correspond, in turn, to particles in the language of the quantum geometry, see, e.g., [?]. Thus, we expect emergence of a non-perturbative scalar field, $\varphi$. Moreover, this field is to be complex and, therefore, the vacuum trajectories, corresponding to the vacuum loops, are to be closed lines. The statement that

$$
\varphi \neq \varphi^{*}
$$

follows from the fact that we started from defects wrapped around a circle, and the directions of the wrapping are topologically distinguishable. It is amusing that a similar $3 \mathrm{~d}$ scalar field was introduced long time ago as the so called thermal scalar, for details and references see, e.g., [?]. Moreover, it seems plausible that the scalar field $\varphi$ associated with the $D 2$ branes considered condenses:

$$
<\varphi>\neq 0 .
$$

In case of the thermal scalar such a condensation was conjectured long ago, for details and references see, e.g., [?, ?]. We will come back to discuss this point further in Section 6.

Coming back to the thermal $D 2$ branes, we note that there is another possible type of such defects. Namely, the branes can be wrapped both around the $x_{4}$ - and $\tau$-circles, with one remaining non-compact coordinate belonging to the ordinary $3 \mathrm{~d}$ space. These objects would also correspond to $3 \mathrm{~d}$ fields. A conspicuous feature of these defect is that they are topologically charged. On the other hand, they are static. Combining these two observations, we conclude that dyons are predicted to exist in the thermal vacuum. Colour electric and magnetic fields are predicted to be equal,

$$
\left|\mathbf{E}^{a}\right|=\left|\mathbf{B}^{a}\right| .
$$

In the field-theoretic language the dyons are described by a doublet of complex scalar fields.

To conclude, there is quite a variety of low-dimensional defects predicted to exist in the YangMills plasma at $T>T_{c}$. It is rather a proliferation of the defects which makes the problem if we try to compare the predictions with the lattice data. Moreover, various defects can merge, for details and references see, e.g., [?]. Thus, we cannot claim that the predictions are already fixed in a unique way. Nevertheless, let us emphasize that the lattice evidence is indeed encouraging. In particular, it is only recently (and unexpectedly) that self-dual dyons, satisfying (??) have been detected on the lattice in the deconfining phase. 


\section{5. "Euclidean superfluidity"}

Let us emphasize again that to apply duality with the stretched horizon one should not consider measurements with poor resolution. It is not for the first time that measurements with poor resolution are invoked to check theory. Indeed, let us recall measurements of the topological susceptibility $\chi$ defined as

$$
\int d^{4} x<G \tilde{G}(x), G \tilde{G}(0)>\chi,
$$

where $G G(x)$ is the density of the topological charge, (see, e.g. [?] and references therein). Since $\chi \sim<Q_{t o p}^{2}>$ the susceptibility is positive definite. At first sight, there is no surprise in this since, say, contribution of instantons to (??) is also positive.

In fact, the condition $\chi>0$ is far from being trivial since for any $x \neq 0$ the sign is just opposite:

$$
<G \tilde{G}(x), G \tilde{G}(0)>_{\text {exact }}<0, \quad x \neq 0 .
$$

This is a consequence of the unitarity (and refers to the Euclidean case). This means that the positive contribution to $\chi$ can only be singular, or of the form:

$$
<G \tilde{G}(x), G \tilde{G}(0)>_{\text {local }}=\delta^{4}(x)\left(\frac{(\text { const }) \alpha_{s}^{2}}{a^{4}}+(\text { const }) \Lambda_{Q C D}^{4}\right)
$$

where $a$ is a cut off. Such a local term cannot be measured directly since it depends on details of definition of the product of operators at coinciding points. The way out is to introduce so called cooling, or suppression of ultraviolet fluctuations. Then the positive term becomes non-local and is determined by instantons. Introduction of cooling corresponds to introducing poor resolution. Note also that cooling is not a unitary deformation of the original fields.

Coming back to the deconfining phase, imagine that we have condensation of a $3 \mathrm{~d}$ complex field. Then there would emerge a massless $3 \mathrm{~d}$ Goldstone. Such a particle would be manifested in the static correlator of momentum densities:

$$
\int d^{3} x \exp \left(i q_{i} r_{i}\right)<T_{0 i}(r), T_{0 k}(0)>\sim \frac{q_{i} q_{k}}{q^{2}} .
$$

Non-vanishing of the coefficient in front of the pole term is a well known signature of superfluidity.

At first sight, we should expect all this to happen if the thermal scalar is condensed. However, the problem is that the thermal scalar behaves as a particle, i.e., its wave function oscillates, in the Euclidean time. Thus, this contribution would contradict unitarity. Optimistically, we can expect to observe correlator of the form (??) in measurements with poor resolution. Such a phenomenon, if observed, could be called "Euclidean superfluidity".

\section{Exotic liquid living on the stretched horizon}

So far, we have been discussing manifestations of condensation of various lower-dimensional defects. We could also apply duality in a different way, assuming that the properties of the liquid living on the stretched horizon, see, e.g., [?], are shared by the gluonic plasma, provided that the measurements are made with poor resolution. Moreover, for large black holes the near-horizon 
space can be approximated by the Rindler space. Within this approximation, a remarkable observation was made recently [?] that the liquid on the stretched horizon in the dissipation-less approximation can be described by a relativistically invariant action. Namely, the action associated with a d-dimensional hypersurface in the $(\mathrm{d}+1)$ dimensional Rindler space takes the form [?]:

$$
S_{\text {liquid }}=T \int d^{d} x \sqrt{-\gamma}
$$

where $T$ is tension, and the action refers to the following metric tensor $\gamma_{a b}(a, b=0,1, . .,(d-1))$

$$
\gamma_{a b} d x^{a} d x^{b} \equiv-r_{c} d \tau^{2}+d x_{i} d x^{i},
$$

where $r_{c}$ is a constant. The relation to the liquid is provided, as usual, by identification of the normalized gradient of the scalar field $\varphi$ with the four-velocity of an ideal liquid $u_{a}$ :

$$
u_{a} \equiv \partial_{a} \varphi / \sqrt{X}, X \equiv-(\partial \varphi)^{2} \equiv\left(\partial_{0} \varphi\right)^{2}-\left(\partial_{i} \varphi\right)^{2}
$$

Finally, the equilibrium solution is given by $\varphi_{\text {equilibrium }}=t$.

For the equilibrium energy-momentum tensor one readily finds:

$$
\left.\left(T_{a b}\right)_{\text {equilibrium }}=-(0, p . ., p)\right), p=1 / \sqrt{r_{c}},
$$

and the pressure $p$ is singular in the limit $r_{c} \rightarrow 0$ (which corresponds to the actual horizon of the black hole). Note that such a form of the equilibrium energy-momentum tensor for the liquid on the horizon is known independently from the membrane paradigm [?].

It is amusing that the action (??) can be independently derived starting from the picture described in Sect. 2, that is, static defects in Euclidean space. The scalar $\varphi$ corresponds then to influctuations of $3 \mathrm{~d}$ volume. Thus, it is no surprise that the form of $\left\langle T_{\mu \nu}>\right.$ given by Eqs. (??) and (??) coincide with each other. What we learn new from Eq. (??) is that the absolute value of the (negative) pressure associated with the non-perturbative physics is to be large. This prediction appears to be in qualitative agreement with the lattice data [?].

\section{References}

[1] E. Witten, "Anti-de Sitter Space, Thermal Phase Transition, And Confinement In Gauge Theories", Adv. Theor. Math. Phys. 2505 (1998), [arXiv:hep-th/9803131].

[2] T. Sakai, Sh. Sugimoto, "Low energy hadron physics in holographic QCD", Prog. Theor. Phys. 113 (2005) 843-882 [arXiv:hep-th/0412141].

[3] V.I. Zakharov, "From confining fields on the lattice to higher dimensions in the continuum", Braz. J. Phys. 37 (2007) 165, arXiv:0612342 [hep-ph]; "Dual string from lattice Yang-Mills theory". AIP Conf. Proc. 756 (2005) 182-191, arXiv:0501011[hep-ph].

[4] A. Gorsky, V. I. Zakharov, "Magnetic strings in lattice QCD as non-Abelian vortices", Phys. Rev. D77 045017 (2008), arXiv:0707.1284 [hep-th]; A. S. Gorsky, V. I. Zakharov, A. R. Zhinitsky, "On classification of QCD defects via holography", Phys. Rev. D79 106003 (2009), arXiv:0902.1842 [hep-ph]. 
[5] E.V. Shuryak, "What RHIC experiments and theory tell us about properties of quark-gluon plasma?" Nucl. Phys. A750 (2005) 64, arXiv: hep-ph/0405066.

[6] O. Aharony, J. Sonnenschein, Sh. Yankielowicz, "A Holographic model of deconfinement and chiral symmetry restoration", Annals Phys. 322 (2007) 1420-1443, arXiv:hep-th/0604161.

[7] H. Verschelde, V.I. Zakharov, "Effective scalar fields in Yang-Mills theories", PoS FACESQCD (2010) 006, arXiv:1107.1393 [hep-th] ; "Long strings and light scalar fields in the quark-gluon plasma", PoS FACESQCD (2010) 041; "Higgs-like scalars from non-perturbative Yang-Mills dynamics", Prog. Theor. Phys. Suppl. 197 (2012) 92-106; Two-component liquid model for the quark-gluon plasma M.N. Chernodub, H. Verschelde, V.I. Zakharov, Theor. Math. Phys. 170 (2012) 211-216, arXiv:1007.1879 [hep-ph].

[8] O. Bergman, G. Lifschytz, "Holographic U(1)(A) and String Creation", JHEP 0704 (2007) 043, arXiv:hep-th/0612289.

[9] Ph. de Forcrand, A. Kurkela, A. Vuorinen, "Center-Symmetric Effective Theory for High-Temperature SU(2) Yang-Mills Theory", Phys. Rev. D77 (2008) 125014 arXiv:0801.1566 [hep-ph]; A. Dumitru, Yun Guo, Y. Hidaka, Ch. P.Korthals Altes, R. D. Pisarski, "How Wide is the Transition to Deconfinement?", Phys. Rev. D83 (2011) 034022 arXiv:1011.3820 [hep-ph].

[10] M. A. Shifman, A.I. Vainshtein, V. I. Zakharov, "QCD and Resonance Physics: Applications", Nucl. Phys. B147 (1979) 448-518.

[11] M.N. Chernodub, A. Nakamura, V.I. Zakharov, "Manifestations of magnetic vortices in equation of state of Yang-Mills plasma", Phys. Rev. D78 (2008) 074021, arXiv:0807.5012 [hep-lat].

[12] A. Di Giacomo, V.I. Zakharov, "Magnetic degrees of freedom in pure Yang-Mills theories", Phys. Atom. Nucl. 73 (2010) 711, arXiv:0806.2938 [hep-th].

[13] M.N. Chernodub, A. D’Alessandro, M. D’Elia , V.I. Zakharov , “Thermal monopoles and selfdual dyons in the Quark-Gluon Plasma", arXiv:0909.5441 [hep-ph] ; V.G. Bornyakov, V.V. Braguta, "Thermal Abelian monopoles, as selfdual dyons", Phys. Rev. D84 (2011) 074502, arXiv:1104.1063 [hep-lat].

[14] J. Ambjorn, "Quantization of geometry", arXiv:hep-th/9411179.

[15] J. J. Atick, E. Witten, 'The Hagedorn Transition and the Number of Degrees of Freedom of String Theory", Nucl. Phys. B310 (1988) 291-334; M. Kruczenski, A. Lawrence, "Random walks and the Hagedorn transition", JHEP 0607 (2006) 031, arXiv:hep-th/0508148.

[16] M. Campostrini, A. Di Giacomo, H. Panagopoulos, "The Topological Susceptibility on the Lattice", Phys. Lett. B212 (1988) 206.

[17] M. Parikh, F. Wilczek, “An Action for black hole membranes", Phys. Rev. D58 (1998) 064011, arXiv:gr-qc/9712077.

[18] I. Bredberg, C. Keeler, V.Lysov, A. Strominger, "Wilsonian Approach to Fluid/Gravity Duality", JHEP 1103 (2011) 141, arXiv:1006.1902 [hep-th]; "From Navier-Stokes To Einstein", JHEP 1207 (2012) 146 arXiv:1101.2451 [hep-th]. 